Теорія Ймовір. та Матем. Статист. Вип. 78, 2008
Theor. Probability and Math. Statist.

No. 78, 2009, Pages 97-114 S 0094-9000(09)00765-0

Article electronically published on August 4, 2009

\title{
DIFFERENCE APPROXIMATION OF THE LOCAL TIMES OF MULTIDIMENSIONAL DIFFUSIONS
}

UDC 519.21

\author{
ALEKSEY M. KULIK
}

\begin{abstract}
Sequences of additive functionals of difference approximations are considered for multidimensional uniformly nondegenerate diffusions. Sufficient conditions are obtained for the weak convergence of such sequences to a $W$-functional of the limit process. The class of $W$-functionals appearing as limits for such a problem can be described uniquely in terms of the corresponding $W$-measures $\mu$ as follows:
\end{abstract}

$$
\begin{aligned}
& \qquad \lim _{\delta \downarrow 0} \sup _{x \in \mathbb{R}^{m}} \int_{\|y-x\| \leq \delta} w(\|y-x\|) \mu(d y)=0, \\
& \text { where } w(r)= \begin{cases}\max (-\ln r, 1), & m=2, \\
r^{2-m}, & m>2 .\end{cases}
\end{aligned}
$$

\section{INTRODUCTION}

We consider a diffusion process $X$ in $\mathbb{R}^{m}$ defined by the following stochastic differential equation

$$
X(t)=X(0)+\int_{0}^{t} a(X(s)) d s+\int_{0}^{t} b(X(s)) d W(s), \quad t \in \mathbb{R}^{+},
$$

and a sequence of processes $X_{n}, n \geq 1$, defined at points $k / n, k \in \mathbb{N}$, by the difference relation

$$
X_{n}\left(\frac{k}{n}\right)=X_{n}\left(\frac{k-1}{n}\right)+a\left(X_{n}\left(\frac{k-1}{n}\right)\right) \cdot \frac{1}{n}+b\left(X_{n}\left(\frac{k-1}{n}\right)\right) \cdot \frac{\xi_{k}}{\sqrt{n}} .
$$

For other points, the $X_{n}$ are defined by linear interpolation; namely,

$$
\begin{gathered}
X_{n}(t)=X_{n}\left(\frac{k-1}{n}\right)+(n t-k+1)\left[X_{n}\left(\frac{k}{n}\right)-X_{n}\left(\frac{k-1}{n}\right)\right], \\
t \in\left[\frac{k-1}{n}, \frac{k}{n}\right) .
\end{gathered}
$$

Here and in what follows the symbol $W$ denotes a Wiener process taking its values in $\mathbb{R}^{d}$, and $\left\{\xi_{k}\right\}$ is a sequence of $\mathbb{R}^{d}$-valued independent identically distributed random vectors belonging to the domain of attraction of the normal distribution. We also assume that the random vectors $\xi_{k}$ are centered and have the unit covariance matrix. Under the standard assumptions on the coefficients of equations (1.1), (1.2) (namely, the local Lipschitz condition and the condition of linear growth), the distributions of the processes

2000 Mathematics Subject Classification. Primary 60J55, 60J45, 60F17.

Key words and phrases. Additive functional, local time, characteristic, $W$-measure, Markov approximation.

Supported by the Ministry of Education and Science of Ukraine, project GP/F13/0095. 
$X_{n}$ with a fixed initial value $X_{n}(0)=x$ weakly converge in $C\left(\mathbb{R}^{+}, \mathbb{R}^{m}\right)$ to the distribution of the process $X$ such that $X(0)=x$ (see [1]).

The following problem is considered in the paper. Let $\left\{\varphi^{s, t}, 0 \leq s \leq t\right\}$ be a $W$ functional of the diffusion process defined by the stochastic differential equation (1.1); that is (see [2], Chapter 6), $\varphi^{s, t}$ is a nonnegative homogeneous additive functional whose characteristic

$$
f^{t}(x) \equiv \mathrm{E}\left[\varphi^{0, t} \mid X(0)=x\right]
$$

is such that $\sup _{x} f^{t}(x)<+\infty, t \in \mathbb{R}^{+}$. We consider a sequence of nonnegative additive functionals $\left\{\varphi_{n}^{s, t}, 0 \leq s \leq t\right\}$ of the processes $X_{n}$ that are of the form

$$
\varphi_{n}^{s, t}=\varphi_{n}^{s, t}\left(X_{n}\right) \stackrel{\text { def }}{=} \frac{1}{n} \sum_{k: s \leq \frac{k}{n}<t} F_{n}\left(X_{n}\left(\frac{k}{n}\right)\right)
$$

and find conditions for the weak convergence of the joint distribution of $\left(\varphi_{n}, X_{n}\right)$ given $X_{n}(0)=x$ to the joint distribution of $(\varphi, X)$ given $X(0)=x$, where $x$ is an arbitrary point of $\mathbb{R}^{m}$.

A motivation for setting such a problem is as follows. It is well known that the theory of additive functionals of Markov processes in $\mathbb{R}^{m}$ is closely related to potential theory. There exists a one-to-one correspondence between $W$-functionals and the so-called $W$ measures (see 2], Chapter 8). Namely, every $W$-functional $\varphi$ can be represented in the following form:

$$
\varphi^{s, t}=\int_{s}^{t} \frac{d \mu}{d \lambda^{m}}(X(r)) d r, \quad 0 \leq s \leq t,
$$

where $\mu$ is the corresponding $W$-measure and $\lambda^{m}$ is the Lebesgue measure in $\mathbb{R}^{m}$. Generally speaking, the $W$-measure $\mu$ is not absolutely continuous with respect to $\lambda^{m}$; the meaning of equality (1.5) for singular $\mu$ can be explained by an approximation of $\mu$ by absolutely continuous measures. The functional $\varphi$ defined by equality (1.5) is called the local time of the process $X$ corresponding to the measure $\mu$.

Using a $W$-functional $\varphi$, one can construct a subprocess $X^{\varphi}$ with the transition probability

$$
\mathrm{P}\left[X^{\varphi}(t) \in \Gamma \mid X^{\varphi}(0)=x\right]=\mathrm{E}\left[\mathbb{1}_{\Gamma}(X(t)) \cdot \exp \left(-\varphi^{0, t}\right) \mid X(0)=x\right],
$$

$t \in \mathbb{R}^{+}, x \in \mathbb{R}^{m}, \Gamma \in \mathfrak{B}\left(\mathbb{R}^{m}\right)$ (see 2], $\S 6$ of Introduction); the measure $\mu$ for this process is treated as the killing measure. Let $X$ be a diffusion process defined by equation (1.1). Its generator is given by

$$
\mathfrak{A}=\sum_{i=1}^{m} a_{i}(x) \frac{\partial}{\partial x_{i}}+\frac{1}{2} \sum_{i, j=1}^{m} \sigma_{i j}(x) \frac{\partial^{2}}{\partial x_{i} \partial x_{j}}, \quad \sigma \equiv\left(\sigma_{i j}\right)_{i, j=1}^{m}=b b^{*} .
$$

We assume that the process $X$ satisfies condition A1) introduced below in Section 2 . This, in particular, implies that the density of the transition probability $p$ exists for the process $X$. If $d \mu / d \lambda^{m}$ is a bounded function, then the well-known Feinman-Kac formula implies that the generator of the process $X^{\varphi}$ is of the form $\mathfrak{A}^{\varphi}=\mathfrak{A}-d \mu / d \lambda^{m}$ (see [2], Theorem 9.7) if $X$ is a Brownian motion. By condition A1), the asymptotic behavior as $t \rightarrow 0+$ of the density of transition probability for the process $X$ is the same as that for the Brownian motion. Thus the result of the mentioned Theorem 9.7 [2] holds for this process, too. This means that, for an arbitrary bounded continuous function $g(\cdot)$, the 
solution of the Cauchy problem for the following second order parabolic equation,

$$
\left\{\begin{aligned}
\frac{\partial}{\partial t} u(t, x)= & \sum_{i=1}^{m} a_{i}(x) \frac{\partial}{\partial x_{i}} u(t, x)+\frac{1}{2} \sum_{i, j=1}^{m} \sigma_{i j}(x) \frac{\partial^{2}}{\partial x_{i} \partial x_{j}} u(t, x) \\
& -\frac{d \mu}{d \lambda^{m}}(x) u(t, x) \\
u(0, x)= & g(x)
\end{aligned}\right.
$$

has the probabilistic representation

$$
u(t, x)=\mathrm{E}\left[g(X(t)) \exp \left(-\varphi^{0, t}\right) \mid X(0)=x\right] .
$$

Now let $d \mu / d \lambda^{m}$ be a generalized function. Moreover let the characteristic

$$
f^{t}(x)=\int_{0}^{t} \int_{\mathbb{R}^{m}} p_{s}(x, y) \mu(d y) d s
$$

of the corresponding additive functional $\varphi$ satisfy the regularity condition:

$$
\sup _{x \in \mathbb{R}^{m}} f^{\delta}(x) \rightarrow 0, \quad \delta \rightarrow 0+
$$

(2], Condition 6.16.A). Then the $W$-functionals $\varphi_{\delta}$ corresponding to $W$-measures $\mu_{\delta}$ with bounded densities $d \mu_{\delta} / d \lambda^{m}=f^{\delta} / \delta$ converge as $\delta \rightarrow 0+$ to $\varphi$ in the mean square sense with respect to the probabilities $\mathrm{P}[\cdot \mid X(0)=x], x \in \mathbb{R}^{m}$; the convergence is uniform in $x \in \mathbb{R}^{m}, s, t \leq T$ (see [2], Theorems 6.4 and 6.6 and Lemma 6.5). Thus

$$
u_{\delta}(t, x) \equiv \mathrm{E}\left[g(X(t)) \exp \left(-\varphi_{\delta}^{0, t}\right) \mid X(0)=x\right] \rightarrow u(t, x), \quad \delta \rightarrow 0+,
$$

uniformly in $x \in \mathbb{R}^{m}, t \leq T$. Moreover the $\mu_{\delta}$ weakly converge to $\mu$. Hence $u(\cdot, \cdot)$ is a continuous function determining the solution of equation (1.7) in a generalized sense:

$$
\begin{aligned}
\int_{\mathbb{R}^{m}}[u(t, x)-g(x)] \psi(x) d x & \\
= & \int_{0}^{t} \int_{\mathbb{R}^{m}}\left[\sum_{i=1}^{m} a_{i} \frac{\partial}{\partial x_{i}} u(s, x)+\frac{1}{2} \sum_{i, j=1}^{m} \sigma_{i j} \frac{\partial^{2}}{\partial x_{i} \partial x_{j}} u(s, x)\right] \psi(x) d x d s \\
& -\int_{0}^{t} \int_{\mathbb{R}^{m}} u(s, x) \psi(x) \mu(d x), \quad \psi \in \mathcal{D}\left(\mathbb{R}^{m}\right),
\end{aligned}
$$

where $\mathcal{D}\left(\mathbb{R}^{m}\right)$ is the Schwartz space of the test functions.

Consider the functionals

$$
u_{n}(t, x)=\mathrm{E}\left[g\left(X_{n}(t)\right) \exp \left(-\varphi_{n}^{0, t}\right) \mid X_{n}(0)=x\right] .
$$

The main result of this paper (Theorem 2.1) provides conditions for the convergence in distribution of a difference approximation $\left(\varphi_{n}, X_{n}\right)$ to $(\varphi, X)$. Thus

$$
u_{n}(t, x) \rightarrow u(t, x), \quad n \rightarrow \infty, \quad t \in \mathbb{R}^{+}, x \in \mathbb{R}^{m},
$$

if the assumptions of Theorem 2.1 hold. In particular, this allows one to apply the Monte Carlo method to solve numerically the Cauchy problem for the parabolic equation (1.7), where the term $d \mu / d \lambda^{m}$ describing the "heat outflow" is a generalized function.

There are many asymptotic results concerning the associated functionals for onedimensional random walks and difference approximations of one-dimensional diffusions [3]-10]. The situation differs essentially in the multidimensional case. The author is unaware of any paper where limit theorems are proved for functionals of the form (1.4) if $X_{n}$ is an approximation of a nonadditive diffusion process $X$ (that is, if the coefficients $a$ and $b$ are not constants). The only multidimensional limit theorem known to the author is obtained in [1] for such functionals if $X_{n}$ is a multidimensional aperiodic lattice random walk and $X$ is the Brownian motion in $\mathbb{R}^{m}$ (related questions are discussed in [12). The essential difference between the existing results in the one-dimensional case and those 
in the multidimensional case is explained by the structure of the $W$-sets, which is much more complicated in the second case. In the case of Brownian motion as well as in the case of an arbitrary nondegenerate diffusion for $m=1$, every finite measure is a $W$-measure. Neither of the measures $\delta_{z}, z \in \mathbb{R}^{m}$, is a $W$-measure for $m>1$ ( $\Leftrightarrow$ there is no local time at any fixed point $\Leftrightarrow$ every singleton has zero capacity $\Leftrightarrow$ every singleton is a polar set). Thus the conditions imposed on the "symbols" $d \mu_{n} / d \lambda^{m} \equiv F_{n}$ of approximating aggregates (1.4) and on the "symbol" $d \mu / d \lambda^{m}$ of the limit functional $\varphi$ are much more subtle. A uniform in $n$ analog of the following condition on the "dimension" of the symbol $d \mu / d \lambda^{m}$ is used in [11]:

$$
\begin{aligned}
& \text { there exist } C \in \mathbb{R}^{+} \text {and } \gamma>m-2 \text { such that } \\
& \mu(\{y:\|y-x\| \leq r\}) \leq C r^{\gamma}, \quad r \geq 0 .
\end{aligned}
$$

The following criterion is well known for the Brownian motion in $\mathbb{R}^{m}: \mu$ is a $W$ measure if and only if

$$
\sup _{x \in \mathbb{R}^{m}} \int_{\|y-x\| \leq 1} w(\|y-x\|) \mu(d y)<+\infty,
$$

where

$$
w(r)= \begin{cases}\max (-\ln r, 1), & m=2, \\ r^{2-m}, & m>2\end{cases}
$$

(see 2, Chapter 8). It is easy to check that condition (1.11) is sufficient for condition (1.12). However it is not necessary (see Example 5.1). This means, in particular, that only functionals of a certain subclass of the family of $W$-functionals of the Brownian motion (namely, the functionals for which the corresponding $W$-measure satisfies the "dimension" condition (1.11) ) may appear as limit functionals in the limit theorem of the paper [11].

The main result of this paper (Theorem 2.1) proves the weak convergence of functionals (1.4) for difference approximations $X_{n}$ of multidimensional uniformly nondegenerate diffusions $X$. The class of difference approximations $X_{n}$ is rather wide in Theorem 2.1, We assume that some modification of the local limit theorem (see Condition B4) in Section 4 below) holds for the densities of transition probabilities of $X_{n}$. The proof of Theorem 2.1 is based on some of the results of 13 and 14 .

The assumption of Theorem 2.1 imposed on the "symbols" of the approximating aggregates is a uniform in $n$ analog of the condition

$$
\lim _{\delta \downarrow 0} \sup _{x \in \mathbb{R}^{m}} \int_{\|y-x\| \leq \delta} w(\|y-x\|) \mu(d y)=0 .
$$

Condition (1.11) is stronger than (1.13) (see Example 5.1). A relationship between conditions (1.13) and (1.12) is discussed below.

In order to make the reasoning shorter we restrict consideration to the case of measures $\mu$ with compact supports. Using standard estimates for the local behavior of the transition density for nonuniform nondegenerate diffusions (see [2], §6 of the Appendix and the references therein) one can check in a standard way for such measures that condition (1.12) is necessary and sufficient for $\mu$ to be a $W$-measure for nonuniformly nondegenerate diffusion. If a measure satisfies (1.13) and has a compact support, then it is finite. Since the function $w(\cdot)$ is bounded on $[\delta,+\infty)$ for an arbitrary $\delta>0$, this implies that the measure $\mu$ satisfies (1.12); that is, it is a $W$-measure.

Properties of characteristics of $W$-functionals whose $W$-measures satisfy (1.13) are given in the following assertion. 
Proposition 1.1. Let $X$ be a diffusion process in $\mathbb{R}^{m}, m \geq 2$, and let condition $\boldsymbol{A} 1$ ) hold for its coefficients. Further let $\mu$ be $a W$-measure with a compact support, $\varphi$ be the $W$-functional corresponding to this measure, and let $f$ be its characteristic. Then the following conditions are equivalent:

(i) for an arbitrary $t \in \mathbb{R}^{+}, f^{t}(\cdot)$ is a uniformly continuous function in $\mathbb{R}^{m}$,

(ii) $\sup _{x \in \mathbb{R}^{m}} f^{\delta}(x) \rightarrow 0, \delta \rightarrow 0+$,

(iii) $\mu$ satisfies condition (1.13).

We prove Proposition 1.1 in Section 4. Proposition 1.1 clarifies condition (1.13); namely, $W$-measures satisfying this condition there correspond to $W$-functionals whose characteristics are continuous functions of two arguments and satisfy the regularity condition (1.9). The class of functionals appearing as limits in the main result of this paper (Theorem 2.1) coincides with the class of functionals that are regular in the sense of Proposition 1.1 (see Remark 2.3 below).

\section{Main Result}

We study the objects defined by relations (1.1)-(1.4) for $m \geq 2$. The Euclidean norm is denoted by $\|\cdot\|$ without an indication of the space which this norm is used for. The class of functions possessing $k$ continuous derivatives is denoted by $C^{k}$, while the class of functions possessing $k$ continuous bounded derivatives is denoted by $C_{b}^{k}$. The weak convergence of finite (not necessarily probability) measures $\mu_{n}$ to a measure $\mu$ is equivalent, by definition, to the convergence of integrals $\int_{\mathbb{R}^{m}} f d \mu_{n} \rightarrow \int_{\mathbb{R}^{m}} f d \mu$ for all $f \in C_{b}\left(\mathbb{R}^{m}\right)$.

Below are the main assumptions imposed on the objects defined by (1.1)-(1.4).

A1) $a \in C_{b}^{2}\left(\mathbb{R}^{m}, \mathbb{R}^{m}\right), b \in C_{b}^{2}\left(\mathbb{R}^{m}, \mathbb{R}^{m \times d}\right)$, and there are positive constants $c$ and $C$ such that

$$
c\|\theta\|^{2} \leq\left(b(x) b^{*}(x) \theta, \theta\right)_{\mathbb{R}^{m}} \leq C\|\theta\|^{2}, \quad x, \theta \in \mathbb{R}^{m} .
$$

Moreover the function $\nabla^{2} b$ satisfies the Hölder condition for some positive index.

A2) Random vectors $\xi_{k}$ are independent identically distributed, centered, and have the unit covariance matrix.

A3) Random vectors $\left\{\xi_{k}\right\}$ have the distribution density $p \in C^{4}\left(\mathbb{R}^{m}\right)$. There exists a function $\psi: \mathbb{R}^{m} \rightarrow \mathbb{R}^{+}$such that

$$
\sup _{x \in \mathbb{R}^{m}} \psi(x)<+\infty, \quad \int_{\mathbb{R}^{m}}\|x\|^{m^{2}+2 m+4} \psi(x) d x<+\infty,
$$

and

$$
\left\|\left[\nabla^{i} p\right](x)\right\| \leq \psi(x), \quad x \in \mathbb{R}^{m}, i=0, \ldots, 4 .
$$

A4) $F_{n}(x) \geq 0, x \in \mathbb{R}^{m}, n \geq 1$, and $n^{-1} \sup _{x \in \mathbb{R}^{m}} F_{n}(x) \rightarrow 0$ as $n \rightarrow \infty$.

A5) Measures $\mu_{n}(d x) \equiv F_{n}(x) \lambda^{m}(d x)$ weakly converge to a finite measure $\mu$ with a compact support.

A6) The following uniform analog of condition (1.13) holds:

$$
\lim _{\delta \downarrow 0} \limsup _{n \rightarrow+\infty} \sup _{x \in \mathbb{R}^{m}} \int_{\|y-x\| \leq \delta} w(\|y-x\|) \mu_{n}(d y)=0 .
$$

Remark 2.1. If conditions $\mathbf{A} 5$ ) and $\mathbf{A} 6$ ) are satisfied, then the measure $\mu$ satisfies condition (1.13); thus $\mu$ is a $W$-measure.

Remark 2.2. The function $w(\cdot)$ is bounded in the interval $[\delta,+\infty)$ for an arbitrary $\delta>0$. Thus conditions A5) and A6) imply that $\lim \sup _{n \rightarrow+\infty} \mu_{n}\left(\mathbb{R}^{m}\right)<+\infty$ and

$$
\limsup _{n \rightarrow+\infty} \sup _{x \in \mathbb{R}^{m}} \int_{\mathbb{R}^{m}} w(\|y-x\|) \mu_{n}(d y)<+\infty .
$$


Now we turn to the main result. Together with functionals $\varphi_{n}$ that are step functions in every time argument, we consider random polygonal lines constructed from these functionals:

$$
\begin{gathered}
\psi_{n}^{s, t}=\varphi_{n}^{\frac{j-1}{n}, \frac{k-1}{n}}+(n s-j+1) \varphi_{n}^{\frac{j-1}{n}, \frac{j}{n}}+(n t-k+1) \varphi_{n^{\frac{k-1}{n}}, \frac{k}{n}}, \\
s \in\left[\frac{j-1}{n}, \frac{j}{n}\right), \quad t \in\left[\frac{k-1}{n}, \frac{k}{n}\right) .
\end{gathered}
$$

We treat these polygonal lines as random elements in the space $C\left(\mathbb{T}, \mathbb{R}^{+}\right)$, where

$$
\mathbb{T} \stackrel{\text { def }}{=}\{(s, t) \mid 0 \leq s \leq t\} .
$$

A $W$-functional $\varphi=\varphi(X)$ of a process $X$ is defined by equality (1.5).

Theorem 2.1. Let conditions $\boldsymbol{A} 1)-\boldsymbol{A} 6)$ hold. Then $\left(X_{n}, \psi_{n}\left(X_{n}\right)\right)$ converge in distribution to $(X, \varphi(X))$ in the space $C\left(\mathbb{R}^{+}, \mathbb{R}^{m}\right) \times C\left(\mathbb{T}, \mathbb{R}^{+}\right)$.

Theorem 2.1 is proved in Section 4.

Remark 2.3. Consider an arbitrary $W$-measure $\mu$ satisfying condition (1.13). One can construct a sequence $\left\{F_{n}\right\}$ of functions such that conditions A4)-A6) hold. As $F_{n}$, one can take, for example, the function

$$
F_{n}(x)=n \int_{0}^{1 / n} \int_{\mathbb{R}^{m}} q_{t}(x, y) \mu(d y) d t, \quad x \in \mathbb{R}^{m},
$$

where

$$
q_{t}(x, y)=(2 \pi t)^{-m / 2} \exp \left(-\frac{\|y-x\|^{2}}{2}\right)
$$

is the density of the transition probability for the Brownian motion $W$. Then $n^{-1} F_{n}$ is the value at the point $1 / n$ of the characteristic of the $W$-functional

$$
L_{t}=\int_{0}^{t} \frac{d \mu}{d \lambda^{m}}\left(W_{s}\right) d s
$$

of the process $W$. Then the proof of conditions A4)-A6) is similar to that of Proposition 1.1 (see Section 4).

\section{WEAK CONVERGENCE OF ADDitive FUnCTIONALS OF A SEQUENCE OF MARKOV CHAINS}

The proof of Theorem 2.1 is based on a general result on the convergence in distribution of a sequence of additive functionals of Markov chains proved in [15. In this section, we describe objects and introduce auxiliary notions necessary to apply this result.

We assume that the processes $X_{n}(\cdot)$ and $X(\cdot)$ are defined on $\mathbb{R}^{+}$and their phase space is a locally compact metric space $(\mathfrak{X}, \rho)$. We say that a process $X$ has the Markov property at a moment $s \in \mathbb{R}^{+}$with respect to the flow of $\sigma$-algebras $\left\{\mathcal{G}_{t}, t \in \mathbb{R}^{+}\right\}$if $X$ is adapted to this flow and, for arbitrary $k \in \mathbb{N}$ and $t_{1}, \ldots, t_{k}>s$, there exists a probability kernel $\left\{P_{s t_{1} \ldots t_{k}}(x, A), x \in \mathfrak{X}, A \in \mathcal{B}\left(\mathfrak{X}^{k}\right)\right\}$ such that

$$
\mathrm{E}\left[\mathbb{1}_{A}\left(\left(X\left(t_{1}\right), \ldots, X\left(t_{k}\right)\right)\right) \mid \mathcal{G}_{s}\right]=P_{s t_{1} \ldots t_{k}}(X(s), A) \quad \text { a.s. }
$$

for all $A \in \mathcal{B}\left(\mathfrak{X}^{k}\right)$. The abbreviation "a.s." means "almost surely". It is natural to consider the measure $P_{s t_{1} \ldots t_{k}}(x, \cdot)$ as the conditional finite-dimensional distribution of $X$ at points $t_{1}, \ldots, t_{k}$ given $\{X(s)=x\}$. Below we use the notation

$$
P_{s t_{1} \ldots t_{k}}(x, \cdot)=\mathrm{P}\left(\left(X\left(t_{1}\right), \ldots, X\left(t_{k}\right)\right) \in \cdot \mid X(s)=x\right) .
$$

Throughout this section we assume that the process $X$ has the Markov property with respect to its canonical flow of $\sigma$-algebras at all points $s \in \mathbb{R}^{+}$and that the processes $X_{n}$ 
have the Markov property at all points $i / n, i \in \mathbb{Z}_{+}$. This means, in fact, that every process $X_{n}$ is a Markov chain in the time scale proportional to $1 / n$.

Let the additive functionals $\varphi_{n}$ be given by equality (1.4). For a functional $\varphi_{n}$, its characteristic $f_{n}$ (which is an analog of the characteristic of a $W$-functional) is defined by

$$
f_{n}^{s, t}(x) \stackrel{\text { def }}{=} \mathrm{E}\left[\varphi_{n}^{s, t}\left(X_{n}\right) \mid X_{n}(s)=x\right], \quad s=\frac{i}{n}, \quad i \in \mathbb{Z}_{+}, t>s, x \in \mathfrak{X} .
$$

Note that the process $X_{n}$ has the Markov property with respect to its canonical flow of $\sigma$ algebras at points $s=i / n, i \in \mathbb{Z}_{+}$, while the functional (1.4) is a function of $X_{n}$ on a finite set of time instances. Thus the mean value in (3.2) is well defined as an integral over a family of conditional finite-dimensional distributions $\left\{P_{s t_{1} \ldots t_{k}}(x, \cdot), t_{1}, \ldots, t_{k}>s, k \in \mathbb{N}\right\}$ of the process $X_{n}$.

To state the general convergence result we need to recall the notion of the Markov approximation introduced in [16].

Definition 3.1. A sequence $\left\{X_{n}\right\}$ approximates a process $X$ in the Markov sense if, for arbitrary $\gamma>0$ and $T<+\infty$, there are a number $K(\gamma, T) \in \mathbb{N}$ and a sequence $\left\{\hat{Y}_{n}=\left(\hat{X}_{n}, \hat{X}^{n}\right)\right\}$ of two component processes, probably defined on a different probability space, such that

(i) $\hat{X}_{n} \stackrel{d}{=} X_{n}, \hat{X}^{n} \stackrel{d}{=} X$;

(ii) the processes $\hat{Y}_{n}, \hat{X}_{n}, \hat{X}^{n}$ have the Markov property at points $i K(\gamma, T) / n, i \in \mathbb{N}$, with respect to the flow $\left\{\hat{\mathcal{F}}_{t}^{n}=\sigma\left(\hat{Y}_{n}(s), s \leq t\right)\right\}$;

$$
\limsup _{n \rightarrow+\infty} \mathrm{P}\left(\sup _{i \leq \frac{T n}{K(\gamma, T)}} \rho\left(\hat{X}_{n}\left(\frac{i K(\gamma, T)}{n}\right), \hat{X}^{n}\left(\frac{i K(\gamma, T)}{n}\right)\right)>\gamma\right)<\gamma .
$$

Theorem 3.1. Let a sequence of processes $X_{n}$ approximate a homogeneous Markov process $X$ in the Markov sense and let $\left\{\varphi_{n} \equiv \varphi_{n}\left(X_{n}\right)\right\}$ be a sequence of functionals of the form (1.4). Assume that

(1) the functions $n^{-1} F_{n}(\cdot)$ are nonnegative, bounded on $\mathfrak{X}$, and uniformly converge to zero:

$$
\delta\left(F_{n}\right) \stackrel{\text { def }}{=} \frac{1}{n} \sup _{x \in \mathcal{X}} F_{n}(x) \rightarrow 0, \quad n \rightarrow \infty,
$$

(2) there exists a function $f$ that is the characteristic of some $W$-functional $\varphi=$ $\varphi(X)$ of the limit Markov process $X$ such that

$$
\sup _{s=i / n, t \in(s, T)} \sup _{x \in \mathfrak{X}}\left|f_{n}^{s, t}(x)-f^{t-s}(x)\right| \rightarrow 0, \quad n \rightarrow \infty,
$$

for an arbitrary $T \in \mathbb{R}^{+}$,

(3) the limit function $f$ is continuous with respect to the argument $x$; moreover, $f$ is uniformly continuous in the time argument, that is,

$$
\sup _{t \leq T}\left|f^{t}(x)-f^{t}(y)\right| \rightarrow 0, \quad\|x-y\| \rightarrow 0,
$$

for all $T$. Then the random polygonal lines $\psi_{n}$ corresponding to the functionals $\varphi_{n}$ converge in distribution in $C\left(\mathbb{T}, \mathbb{R}^{+}\right)$:

$$
\psi_{n}\left(X_{n}\right) \Rightarrow \varphi(X) \equiv\left\{\varphi^{s, t}(X),(s, t) \in \mathbb{T}\right\} .
$$

In addition, if $X_{n} \Rightarrow X$ in $C\left(\mathbb{R}^{+}, \mathfrak{X}\right)$, then $\left(X_{n}, \psi_{n}\left(X_{n}\right)\right) \Rightarrow(X, \varphi(X))$ in the space $C\left(\mathbb{R}^{+}, \mathfrak{X}\right) \times C\left(\mathbb{T}, \mathbb{R}^{+}\right)$. 
The result of Theorem 3.1 is proved in [15. (Theorem 1 and Remark 5). This result is an analog of a well-known Dynkin theorem expressing the convergence of $W$-functionals in terms of their characteristics ([2, Theorem 6.4).

The following auxiliary result explains Definition 3.1 It also helps to apply Theorem 3.1 in the proof of the main result of this paper.

Lemma 3.1. Let $a$ and $b$ be bounded and satisfy the Lipschitz condition, let $m \geq 1$ be an arbitrary number, and let independent identically distributed random vectors $\xi_{k}$ be centered, have the unit covariance matrix, and be such that $\mathrm{E}\left\|\xi_{k}\right\|^{2+\delta}<+\infty$ for some $\delta>0$.

Then the sequence of processes $X_{n}$ defined by equalities (1.2) and (1.3) approximates the process $X$ defined by equation (1.1) in the Markov sense.

Proof. The lemma is proved in Example 3 of [15] by using the uniqueness of the pathwise solution of equation (1.1). This method does not allow one to obtain the rate of convergence. Below we provide a direct proof useful for further generalizations and for obtaining the explicit bounds.

We start with the construction used in the proof of Theorem 1 in [16]. Let

$$
S_{N}=\sum_{k=1}^{N} \xi_{k}
$$

By the central limit theorem, $n^{-1 / 2} S_{n} \Rightarrow W(1)$. The condition $\mathrm{E}\left\|\xi_{k}\right\|^{2+\delta}<+\infty$ implies that the family $\left\{S_{N}^{2} / N\right\}$ is uniformly integrable, whence we get that the Wasserstein distance (see, for example, [17]) between the distributions of the vectors $N^{-1 / 2} S_{N}$ and $W(1)$ approaches 0 as $N \rightarrow \infty$. This means that, given an arbitrary $\varepsilon>0$, there are $N_{\varepsilon} \in \mathbb{N}$ and a random vector $\left(\eta_{\varepsilon}, \zeta_{\varepsilon}\right)$ such that

$$
\mathrm{E}\left\|\eta_{\varepsilon}-\zeta_{\varepsilon}\right\|_{\mathbb{R}^{m}}^{2}<\varepsilon, \quad \eta_{\varepsilon} \stackrel{\mathrm{d}}{=} \frac{S_{N_{\varepsilon}}}{\sqrt{N_{\varepsilon}}}, \quad \zeta_{\varepsilon} \stackrel{\mathrm{d}}{=} W(1)
$$

Fix $\varepsilon>0$ and put $\Omega^{1}=\left(\mathbb{R}^{m}\right)^{N_{\varepsilon}} \times C([0,1])$ and $\mathcal{F}^{1}=\mathcal{B}\left(\Omega^{1}\right)$. Denote the coordinates of the point $\omega^{1} \in \Omega^{1}$ by $\chi=\left(\chi_{1}, \ldots, \chi_{N_{\varepsilon}}\right) \in\left(\mathbb{R}^{m}\right)^{N_{\varepsilon}}, \varpi \in C([0,1])$. Let $Q(d u, d v)$ be the joint distribution of $\left(\eta_{\varepsilon}, \zeta_{\varepsilon}\right)$, let $U_{\varepsilon}(d \chi, u)$ be the conditional distribution of $\left\{\xi_{1}, \ldots, \xi_{N_{\varepsilon}}\right\}$ given $\left\{S_{N_{\varepsilon}} / \sqrt{N_{\varepsilon}}=u\right\}$, and let $V_{\varepsilon}(d \varpi, v)$ be the conditional distribution of $W(\cdot)$ given $\{W(1)=v\}$. Put

$$
P^{1}(A)=\int_{\left(\mathbb{R}^{m}\right)^{2}}\left[\int_{A} U_{\varepsilon}(d \chi, u) V_{\varepsilon}(d \varpi, v)\right] Q(d u, d v), \quad A \in \mathcal{F}^{1} .
$$

Now we introduce the probability space $(\Omega, \mathcal{F}, \mathrm{P})$ as the countable product of copies of the space $\left(\Omega^{1}, \mathcal{F}^{1}, P^{1}\right)$. For $\omega=\left(\chi^{1}, \varpi^{1}, \chi^{2}, \varpi^{2}, \ldots\right) \in \Omega$, define the sequence

$$
\left\{\hat{\xi}_{k}(\omega), k \geq 1\right\}
$$

by

$$
\hat{\xi}_{1}(\omega)=\chi_{1}^{1}, \quad \hat{\xi}_{2}(\omega)=\chi_{2}^{1}, \quad \ldots, \quad \hat{\xi}_{N_{\varepsilon}}(\omega)=\chi_{N_{\varepsilon}}^{1}, \quad \xi_{N_{\varepsilon}+1}(\omega)=\chi_{1}^{2}, \quad \ldots,
$$

and the process $\left\{\hat{W}^{n}(t), t \in \mathbb{R}^{+}\right\}$by

$$
\hat{W}^{n}(t)(\omega)=\frac{1}{\sqrt{n}}\left[\sum_{k=1}^{[n t]} \varpi^{k}(1)+\varpi^{[n t]+1}(n t-[n t])\right], \quad t \geq 0 .
$$


By definition, the sequence $\left\{\hat{\xi}_{k}\right\}$ has the same distribution as the sequence $\left\{\xi_{k}\right\}$ does. Again by definition, the process

$$
\Lambda_{n}^{k}:\left[\frac{k-1}{n}, \frac{k}{n}\right) \ni t \mapsto \frac{1}{\sqrt{n}} \varpi^{k}\left(n t-\frac{k-1}{n}\right)
$$

has the same distribution as the process

$$
\left[\frac{k-1}{n}, \frac{k}{n}\right) \ni t \mapsto W(t)-W\left(\frac{k-1}{n}\right)
$$

does. Since the processes $\left\{\Lambda_{n}^{k}, k \geq 1\right\}$ are jointly independent, the process $\hat{W}^{n}$ is a Brownian motion in $\mathbb{R}^{m}$.

Now we construct processes $\hat{X}_{n}$ according to equalities (1.2) and (1.3), where the sequence $\left\{\xi_{k}\right\}$ is changed for $\left\{\hat{\xi}_{k}\right\}$. The processes $\hat{X}^{n}$ are constructed according to (1.1), where $W$ is changed for $\hat{W}^{n}$. By construction, condition (i) of Definition 3.1 holds for the process $\hat{Y}_{n}=\left(\hat{X}_{n}, \hat{X}^{n}\right)$.

Further, the families

$$
\Xi_{n}^{l}=\left\{\hat{\xi}_{l N_{\varepsilon}+1}, \ldots, \hat{\xi}_{(l+1) N_{\varepsilon}},\left.\left(\hat{W}^{n}(\cdot)-\hat{W}^{n}\left(\frac{l N_{\varepsilon}}{n}\right)\right)\right|_{\left[\frac{l N_{\varepsilon}}{n}, \frac{(l+1) N_{\varepsilon}}{n}\right]}\right\}, \quad l=0,1, \ldots,
$$

are jointly independent by construction. Considering (1.1)-1.3), the values of the process $\hat{Y}_{n}$ at moments $i N_{\varepsilon} / n, i \in \mathbb{N}$, are a functional of $\Xi_{n}^{0}, \ldots, \Xi_{n}^{i-1}$. Similarly the values of $\hat{Y}_{n}$, $\hat{X}_{n}$, or $\hat{X}^{n}$ at every time instance $t>i N_{\varepsilon} / n$ are functionals of $\Xi_{n}^{i}, \Xi_{n}^{i+1}, \ldots$ and also of $\hat{Y}_{n}\left(i N_{\varepsilon} / n\right)$ and $\hat{X}_{n}\left(i N_{\varepsilon} / n\right)$, or $\hat{X}^{n}\left(i N_{\varepsilon} / n\right)$, respectively. Thus the processes $\hat{Y}_{n}, \hat{X}_{n}$, and $\hat{X}^{n}$ have the Markov property with respect to the flow $\left\{\hat{\mathcal{F}}_{t}, t \in \mathbb{R}^{+}\right\}$generated by $\hat{Y}_{n}$ at the time instances $i N_{\varepsilon} / n, i \in \mathbb{N}$.

Now we estimate the distance between $\hat{X}_{n}$ and $\hat{X}^{n}$. We restrict consideration to the case of $m=1$ and $a \equiv 0$. The reasoning is the same in the general case but the notation is more complicated. Put

$$
\begin{gathered}
t_{i}=\frac{i N_{\varepsilon}}{n}, \quad \Delta_{i, n}=\hat{X}_{n}\left(t_{i}\right)-\hat{X}_{n}\left(t_{i-1}\right)=\sum_{l=1}^{N_{\varepsilon}} b\left(\hat{X}_{n}\left(t_{i-1}+\frac{l-1}{n}\right)\right) \frac{\hat{\xi}_{(i-1) N_{\varepsilon}+l}}{\sqrt{n}}, \\
\Delta_{i}^{n}=\hat{X}^{n}\left(t_{i}\right)-\hat{X}^{n}\left(t_{i-1}\right)=\int_{t_{i-1}}^{t_{i}} b\left(\hat{X}^{n}(s)\right) d W^{n}(s), \quad i \in \mathbb{N} .
\end{gathered}
$$

By construction, $\mathrm{E}\left[\Delta_{i, n} \mid \hat{\mathcal{F}}_{t_{i-1}}\right]=\mathrm{E}\left[\Delta_{i}^{n} \mid \hat{\mathcal{F}}_{t_{i-1}}\right]=0$, whence

$$
\mathrm{E}\left(\hat{X}_{n}\left(t_{i}\right)-\hat{X}^{n}\left(t_{i}\right)\right)^{2}=\mathrm{E}\left(\hat{X}_{n}\left(t_{i-1}\right)-\hat{X}^{n}\left(t_{i-1}\right)\right)^{2}+\mathrm{E}\left(\Delta_{i, n}-\Delta_{i}^{n}\right)^{2}, \quad i \in \mathbb{N} .
$$

Consider the expansion

$$
\Delta_{i, n}=b\left(\hat{X}_{n}\left(t_{i-1}\right)\right) \sum_{l=1}^{N_{\varepsilon}} \frac{\hat{\xi}_{(i-1) N_{\varepsilon}+l}}{\sqrt{n}}+\Gamma_{i, n}
$$

where

$$
\Gamma_{i, n}=\sum_{l=1}^{N_{\varepsilon}}\left[b\left(\hat{X}_{n}\left(t_{i-1}+\frac{l-1}{n}\right)\right)-b\left(\hat{X}_{n}\left(t_{i-1}\right)\right)\right] \frac{\hat{\xi}_{(i-1) N_{\varepsilon}+l}}{\sqrt{n}} .
$$

The pair of processes $\left(\left\{\hat{X}_{n}(t), t \in \mathbb{R}^{+}\right\},\left\{\hat{\xi}_{k}, k \in \mathbb{N}\right\}\right)$ has the same distribution as the pair

$$
\left(\left\{X_{n}(t), t \in \mathbb{R}^{+}\right\},\left\{\xi_{k}, k \in \mathbb{N}\right\}\right) .
$$


Thus, for all $k \geq 1$, the random variable $\hat{\xi}_{k}$ is independent of the values of the process $\hat{X}_{n}$ on the interval $[0,(k-1) / n]$. Since the function $b$ is bounded by the constant $B$ and satisfies Lipschitz's condition with the constant $L$, we obtain the upper bound

$$
\begin{aligned}
\mathrm{E} \Gamma_{i, n}^{2} & \leq \frac{L^{2}}{n} \sum_{l=1}^{N_{\varepsilon}} \mathrm{E}\left[\hat{X}_{n}\left(t_{i-1}+\frac{l-1}{n}\right)-\hat{X}_{n}\left(t_{i-1}\right)\right]^{2} \leq \frac{L^{2}}{n} \sum_{l=1}^{N_{\varepsilon}} B^{2} \frac{l-1}{n} \\
& <\frac{L^{2} B^{2} N_{\varepsilon}^{2}}{2 n^{2}} .
\end{aligned}
$$

We also consider the following expansion:

$$
\Delta_{i}^{n}=b\left(\hat{X}^{n}\left(t_{i-1}\right)\right)\left[\hat{W}^{n}\left(t_{i}\right)-\hat{W}^{n}\left(t_{i-1}\right)\right]+\Gamma_{i}^{n},
$$

where the second term $\Gamma_{i}^{n}$ (we do not write it explicitly) is estimated in the same way as in (3.4):

$$
\mathrm{E}\left[\Gamma_{i}^{n}\right]^{2} \leq \frac{L^{2} B^{2} N_{\varepsilon}^{2}}{2 n^{2}}
$$

Finally we consider

$$
\begin{aligned}
& b\left(\hat{X}_{n}\left(t_{i-1}\right)\right) \sum_{l=1}^{N_{\varepsilon}} \frac{\hat{\xi}_{(i-1) N_{\varepsilon}+l}}{\sqrt{n}}-b\left(\hat{X}^{n}\left(t_{i-1}\right)\right)\left[\hat{W}^{n}\left(t_{i}\right)-\hat{W}^{n}\left(t_{i-1}\right)\right] \\
& =\left[b\left(\hat{X}_{n}\left(t_{i-1}\right)\right)-b\left(\hat{X}^{n}\left(t_{i-1}\right)\right)\right]\left[\hat{W}^{n}\left(t_{i}\right)-\hat{W}^{n}\left(t_{i-1}\right)\right]+\sqrt{\frac{N_{\varepsilon}}{n}} b\left(\hat{X}_{n}\left(t_{i-1}\right)\right) \Upsilon_{n}^{i},
\end{aligned}
$$

where

$$
\Upsilon_{n}^{i}=\frac{1}{\sqrt{N_{\varepsilon}}} \sum_{l=1}^{N_{\varepsilon}} \hat{\xi}_{(i-1) N_{\varepsilon}+l}-\left[t_{i}-t_{i-1}\right]^{-1 / 2}\left[\hat{W}^{n}\left(t_{i}\right)-\hat{W}^{n}\left(t_{i-1}\right)\right] .
$$

By construction, the random variables $\left[\hat{W}^{n}\left(t_{i}\right)-\hat{W}^{n}\left(t_{i-1}\right)\right]$ and $\Upsilon_{n}^{i}$ are independent of the values of the process $\hat{Y}_{n}$ on the interval $\left[0,(i-1) N_{\varepsilon} / n\right]$ whatever $i \geq 1$ is. Moreover, $\mathrm{E}\left[\Upsilon_{n}^{i}\right]^{2}<\varepsilon$. Thus

$$
\begin{gathered}
\mathrm{E}\left[b\left(\hat{X}_{n}\left(t_{i-1}\right)\right)-b\left(\hat{X}^{n}\left(t_{i-1}\right)\right)\right]^{2}\left[\hat{W}^{n}\left(t_{i}\right)-\hat{W}^{n}\left(t_{i-1}\right)\right]^{2} \\
\leq \frac{L^{2} N_{\varepsilon}}{n} \mathrm{E}\left(\hat{X}_{n}\left(t_{i-1}\right)-\hat{X}^{n}\left(t_{i-1}\right)\right)^{2} \\
\mathrm{E}\left[b\left(\hat{X}_{n}\left(t_{i-1}\right)\right) \Upsilon_{n}^{i}\right]^{2} \leq B^{2} \varepsilon^{2} .
\end{gathered}
$$

We have

$$
\begin{aligned}
\left|\Delta_{i, n}-\Delta_{i}^{n}\right| \leq & \Gamma_{i, n}+\Gamma_{i}^{n}+\left|b\left(\hat{X}_{n}\left(t_{i-1}\right)\right)-b\left(\hat{X}^{n}\left(t_{i-1}\right)\right)\right|\left|\hat{W}^{n}\left(t_{i}\right)-\hat{W}^{n}\left(t_{i-1}\right)\right| \\
& +\left|b\left(\hat{X}_{n}\left(t_{i-1}\right)\right) \Upsilon_{n}^{i}\right| .
\end{aligned}
$$

Note that the bound for the second moment is already obtained for each of the four terms on the right hand side of this inequality (see estimates (3.4)-(3.7)). Now we derive from (3.3) and the Cauchy inequality $\left(\sum_{k \leq 4} x_{k}\right)^{2} \leq 4 \sum_{k \leq 4} x_{k}^{2}$ that

$$
\begin{aligned}
\mathrm{E}\left(\hat{X}_{n}\left(t_{i}\right)-\hat{X}^{n}\left(t_{i}\right)\right)^{2} \leq & \left(1+\frac{4 L^{2} N_{\varepsilon}}{n}\right) \mathrm{E}\left(\hat{X}_{n}\left(t_{i-1}\right)-\hat{X}^{n}\left(t_{i-1}\right)\right)^{2} \\
& +\frac{4 L^{2} B^{2} N_{\varepsilon}^{2}}{n^{2}}+\frac{4 B^{2} \varepsilon N_{\varepsilon}}{n} .
\end{aligned}
$$


Iterating (3.8) we get

$$
\mathrm{E}\left(\hat{X}_{n}\left(t_{i}\right)-\hat{X}^{n}\left(t_{i}\right)\right)^{2} \leq\left[\frac{4 L^{2} B^{2} N_{\varepsilon}}{n}+4 B^{2} \varepsilon\right] \frac{N_{\varepsilon}}{n} \sum_{j \leq i}\left(1+\frac{4 L^{2} N_{\varepsilon}}{n}\right)^{j} .
$$

Let $t_{i} \leq T$, that is, $i \leq T n / N_{\varepsilon}$. Then the number of terms in the sum on the right hand side of (3.9) does not exceed $T n / N_{\varepsilon}$ and each of them does not exceed

$$
\exp \left[\frac{4 L^{2} N_{\varepsilon}}{n} \frac{T n}{N_{\varepsilon}}\right]=e^{4 L^{2} T} .
$$

Finally

$$
\mathrm{E}\left(\hat{X}_{n}\left(t_{i}\right)-\hat{X}^{n}\left(t_{i}\right)\right)^{2} \leq\left[\frac{4 L^{2} B^{2} N_{\varepsilon} T}{n}+4 B^{2} T \varepsilon\right] e^{4 L^{2} T} .
$$

The sequence $\left\{\left(\hat{X}_{n}\left(t_{i}\right)-\hat{X}^{n}\left(t_{i}\right)\right), i \in \mathbb{N}\right\}$ is a martingale by construction. Applying the maximal inequality for martingales $([18$, Chapter VII, $\S 3)$ we get the following estimate:

$$
\begin{aligned}
\mathrm{P}\left[\max _{i: t_{i} \leq T}\left(\hat{X}_{n}\left(t_{i}\right)-\hat{X}^{n}\left(t_{i}\right)\right)^{2} \geq \gamma^{2}\right] & \leq \gamma^{-2}\left[\frac{4 L^{2} B^{2} N_{\varepsilon} T}{n}+4 B^{2} T \varepsilon\right] e^{4 L^{2} T}, \\
\gamma & >0 .
\end{aligned}
$$

Now we can complete the proof of the lemma. Given $\gamma$ and $T$ we choose $\varepsilon>0$ such that

$$
16 B^{2} T e^{4 L^{2} T} \varepsilon<\gamma^{3}
$$

and use this $\varepsilon$ in the above construction. As we have seen, this construction is such that conditions (i) and (ii) hold for $K(\gamma, T)=N_{\varepsilon}$. Bound (3.11) implies condition (iii). The lemma is proved.

Remark 3.1. Denote by $\mathbf{K}(\gamma, T)$ the minimal $K \in \mathbb{N}$ such that there exists a process $\hat{Y}_{n}$ satisfying conditions (i)-(iii) of Definition 3.1 with $K(\gamma, T)=K$. It is shown in [16 (part II of Theorem 1) that

$$
\mathbf{K}(\gamma, T) \rightarrow 0, \quad \gamma \rightarrow 0+,
$$

for the case of $a \equiv 0$ and $b \equiv I_{\mathbb{R}^{m}}$ if the distribution of $\xi_{1}$ is non-Gaussian. In other words, the better the accuracy of the approximation, the worse the Markov properties of the two-component process $\hat{Y}_{n}$ are. The accuracy of the approximation is described by the parameter $\gamma$, while the Markov properties are described by $\mathbf{K}(\gamma, T)$.

\section{Proof of Theorem 2.1 and Proposition 1.1}

We check the assumptions of Theorem 3.1 to prove Theorem 2.1. The sequence $X_{n}$ approximates the process $X$ in the Markov sense by Lemma 3.1. Assumption (1) of Theorem 3.1 follows from condition A4). Now we check assumptions (2) and (3) of Theorem 3.1. The characteristic $f^{t}$ of the functional

is of the form

$$
\varphi^{s, t}=\int_{s}^{t} \frac{d \mu}{d \lambda^{m}}(X(r)) d r
$$

$$
f^{t}(x)=\int_{0}^{t} \int_{\mathbb{R}^{m}} p_{r}(x, y) \mu(d y) d r, \quad x \in \mathbb{R}^{m}, t \geq 0,
$$

where $\left\{p_{t}(x, y), t \geq 0, x, y \in \mathbb{R}^{m}\right\}$ is the density of the transition probability of the process $X$. The existence of such a density is a classical result of the theory of parabolic equations under condition A1). Moreover, the density possesses the following properties (see, for example, $\S 6$ of Appendix in 2 and the references therein). 
B1) For all $0<\delta<T$, the function $p:(t, x, y) \mapsto p_{t}(x, y)$ is uniformly continuous on $[\delta, T] \times \mathbb{R}^{m} \times \mathbb{R}^{m}$.

B2) There are constants $M, \alpha>0$ such that

$$
p_{t}(x, y) \leq M t^{-m / 2} \exp \left(-\frac{\alpha\|y-x\|^{2}}{t}\right) .
$$

B3) There are constants $M_{1}, M_{2}, \alpha, \beta, \lambda>0$ such that

$$
p_{t}(x, y) \geq M_{1} t^{-m / 2} \exp \left(-\frac{\alpha\|y-x\|^{2}}{t}\right)-M_{2} t^{-m / 2+\lambda} \exp \left(-\frac{\beta\|y-x\|^{2}}{t}\right) .
$$

If conditions A2) and A3) hold, then the processes $X_{n}$ have the densities of the transition probabilities $p_{t}^{n}(x, y)$ at the time instances $t \in n^{-1} \mathbb{N}$, that is,

$$
\begin{gathered}
\mathrm{P}\left(X_{n}(t) \in \Gamma \mid X_{n}(s)=x\right)=\int_{\Gamma} p_{t-s}^{n}(x, y) d y, \\
s, t \in \frac{1}{n} \mathbb{Z}, s<t, \quad x \in \mathbb{R}^{m}, \Gamma \in \mathfrak{B}\left(\mathbb{R}^{m}\right) .
\end{gathered}
$$

Moreover, the function $p_{t}^{n}$ is continuous for all $t \in n^{-1} \mathbb{N}$. The characteristics $f_{n}$ of the functionals $\varphi_{n}$ can be expressed in terms of the densities by

$$
\begin{aligned}
f_{n}^{s, t}(x) & =\frac{1}{n} F_{n}(x)+\frac{1}{n} \sum_{k \in \mathbb{N}, k / n<t-s} \int_{\mathbb{R}^{m}} F_{n}(y) p_{k / n}^{n}(x, y) d y \\
& =f_{n}^{0, t-s}(x), \quad s \leq t, x \in \mathbb{R}^{m} .
\end{aligned}
$$

Let $f_{n}^{0, t}=f_{n}^{t}$. According to Theorem 2.1 of [13] and Theorem 1 of [14], conditions A1)A3) imply the following bound for the deviation of the density $p_{t}^{n}$ from the density $p_{t}$.

B4) For all $T \in \mathbb{R}^{+}$,

$$
\sup _{n \in \mathbb{N}, t \leq T} \sup _{x, y \in \mathbb{R}^{m}} \sqrt{n} t^{-m / 2}\left(1+\left(\frac{\|y-x\|}{\sqrt{t}}\right)^{m}\right)\left|p_{t}(x, y)-p_{t}^{n}(x, y)\right|<+\infty .
$$

For $\delta>0$, put

$$
\begin{gathered}
f_{\delta}^{t}(x)=f^{t}(x)-f^{\delta \wedge t}(x)=\int_{\delta \wedge t}^{t} \int_{\mathbb{R}^{m}} p_{r}(x, y) \mu(d y) d r, \quad x \in \mathbb{R}^{m}, t \geq 0, \\
f_{n, \delta}^{t}(x)=f_{n}^{t}(x)-f_{n}^{\delta \wedge t}(x)=\frac{1}{n} \sum_{k \in \mathbb{N}, \delta \leq k / n<t} \int_{\mathbb{R}^{m}} F_{n}(y) p_{k / n}^{n}(x, y) d y, \quad s \leq t, x \in \mathbb{R}^{m} .
\end{gathered}
$$

Conditions A4), A5), and properties B1), B2), and B4) imply the following result. Its proof is standard (see, for example, the reasoning in the proof of Theorem 2 of [15]); thus we omit it.

Proposition 4.1. For an arbitrary $\delta>0$,

(i) the function $f_{\delta}:(t, x) \mapsto f_{\delta}^{t}(x)$ is uniformly continuous on $[0, T] \times \mathbb{R}^{m}$,

(ii) the functions $f_{n, \delta}:(t, x) \mapsto f_{n, \delta}^{t}(x)$ converge as $n \rightarrow \infty$ to the function $f_{\delta}$ uniformly on $[0, T] \times \mathbb{R}^{m}$.

Proposition 4.1 implies that assumptions (2) and (3) of Theorem 3.1 follow from

$$
\begin{gathered}
\lim _{\delta \downarrow 0} \sup _{x \in \mathbb{R}^{m}} f^{\delta}(x)=0, \\
\lim _{\delta \downarrow 0} \limsup _{n \rightarrow+\infty} \sup _{x \in \mathbb{R}^{m}} f_{n}^{\delta}(x)=0 .
\end{gathered}
$$

Thus Theorem 2.1 follows from (4.2)- - too. 
We prove (4.3). Properties B2) and B4) imply that, for an arbitrary $T \in \mathbb{R}^{+}$, there exists a constant $C_{T} \in \mathbb{R}^{+}$such that

$$
p_{t}^{n}(x, y) \leq C_{T} t^{-m / 2}\left[\exp \left(-\frac{\alpha\|y-x\|^{2}}{t}\right)+\left(1+\left(\frac{\|y-x\|}{\sqrt{t}}\right)^{m}\right)^{-1}\right]
$$

for $t \in n^{-1} \mathbb{N}, t \leq T, n \in \mathbb{N}$. Then relation (4.1) yields the bound

$$
\sup _{x \in \mathbb{R}^{m}} f_{n}^{\delta}(x) \leq \frac{1}{n} \sup _{x \in \mathbb{R}^{m}} F_{n}(x)+\sup _{x \in \mathbb{R}^{m}} \int_{\mathbb{R}^{m}} K_{n, \delta}(\|y-x\|) \mu_{n}(d y)
$$

for $\delta \leq 1$, where

$$
K_{n, \delta}(z)=\frac{C_{1}}{n} \sum_{k=1}^{[n \delta]}\left(\frac{n}{k}\right)^{m / 2}\left[\exp \left(-\alpha z^{2} \frac{n}{k}\right)+\left(1+\left(z \sqrt{\frac{n}{k}}\right)^{m}\right)^{-1}\right], \quad z \in \mathbb{R}^{+} .
$$

For all $k \in \mathbb{N}$ and $t \in[k / n,(k+1) / n]$,

$$
\begin{gathered}
\left(\frac{n}{k}\right)^{m / 2} \leq\left(\frac{k+1}{k}\right)^{m / 2} t^{-m / 2} \leq 2^{m / 2} t^{-m / 2} \\
\exp \left(-\alpha z^{2} \frac{n}{k}\right)+\left(1+\left(z \sqrt{\frac{n}{k}}\right)^{m}\right)^{-1} \leq \exp \left(-\frac{\alpha z^{2}}{t}\right)+\left(1+\left(\frac{z}{\sqrt{t}}\right)^{m}\right)^{-1},
\end{gathered}
$$

whence

$$
\begin{aligned}
& \frac{1}{n}\left(\frac{n}{k}\right)^{m / 2}\left[\exp \left(-\alpha z^{2} \frac{n}{k}\right)+\left(1+\left(z \sqrt{\frac{n}{k}}\right)^{m}\right)^{-1}\right] \\
& \leq 2^{m / 2} \int_{k / n}^{(k+1) / n} t^{-m / 2}\left[\exp \left(-\frac{\alpha z^{2}}{t}\right)+\left(1+\left(\frac{z}{\sqrt{t}}\right)^{m}\right)^{-1}\right] d t
\end{aligned}
$$

for all $k \in \mathbb{N}$. An estimate for the kernel $K_{n, \delta}$ follows from (4.5):

$$
\begin{aligned}
K_{n, \delta}(z) & \leq 2^{m / 2} C_{1} \int_{1 / n}^{\delta} t^{-m / 2}\left[\exp \left(-\frac{\alpha z^{2}}{t}\right)+\left(1+\left(\frac{z}{\sqrt{t}}\right)^{m}\right)^{-1}\right] d t \\
& \leq K_{\delta}(z) \equiv 2^{m / 2} C_{1} \int_{0}^{\delta} t^{-m / 2}\left[\exp \left(-\frac{\alpha z^{2}}{t}\right)+\left(1+\left(\frac{z}{\sqrt{t}}\right)^{m}\right)^{-1}\right] d t .
\end{aligned}
$$

The kernel $K_{\delta}$ is estimated in a way similar to that used in [2], §6.1. Changing the variables $u=z^{2} / t$ we get the representations

$$
\begin{gathered}
K_{\delta}(z)=2^{m / 2} C_{1} z^{2-m} G\left(\frac{z^{2}}{\delta}\right), \\
G(y)=\int_{y}^{\infty} u^{m / 2-2}\left[\exp (-\alpha u)+\left(1+u^{m / 2}\right)^{-1}\right] d u .
\end{gathered}
$$

The following properties of the function $G$ can be checked explicitly:

$$
\lim _{y \rightarrow+\infty} G(y)=0, \quad \begin{cases}G(\cdot) \text { is bounded, } & m>2, \\ G(y) \sim-2 \ln y \text { as } y \rightarrow 0+, & m=2 .\end{cases}
$$

Below are two necessary bounds. First,

$$
K_{\delta}(z) \leq D w(z) G\left(\delta^{-1 / 2}\right), \quad z>\delta^{1 / 4}
$$

(here and in what follows we denote by $D$ a positive constant whose precise value does not matter for our purposes; moreover $D$ may denote different constants in different places). 
Bound (4.8) follows directly from (4.6) if $m>2$; for $m=2$, one should additionally use the inequality $w(z) \geq 1$ to prove (4.8). Second,

$$
K_{\delta}(z) \leq D w(z), \quad z \in \mathbb{R}^{+}, \delta \leq 1 .
$$

Bound (4.9) follows from the boundedness of the function $G$ if $m=3$. If $m=2$, then it follows from

$$
G(y) \leq D w(y), \quad G\left(\frac{z^{2}}{\delta}\right) \leq D w\left(\frac{z^{2}}{\delta}\right) \leq D w\left(z^{2}\right) \leq 2 D w(z)
$$

(we used the restriction $\delta \leq 1$ ). Relation (4.4), condition A4), and bounds (4.8) and (4.9) yield

$$
\begin{aligned}
\limsup _{n \rightarrow+\infty} \sup _{x \in \mathbb{R}^{m}} f_{n}^{\delta}(x) \leq & D G\left(\delta^{-1 / 2}\right) \limsup _{n \rightarrow+\infty} \sup _{x \in \mathbb{R}^{m}} \int_{\mathbb{R}^{m}} w(\|y-x\|) \mu_{n}(d y) \\
& +D \limsup _{n \rightarrow+\infty} \sup _{x \in \mathbb{R}^{m}} \int_{\|y-x\|<\delta^{1 / 4}} w(\|y-x\|) \mu_{n}(d y) .
\end{aligned}
$$

Both terms on the right hand side of (4.10) approach zero as $\delta \downarrow 0$ in view of (4.7), Remark 2.2. and condition A6). Thus relation (4.3) is proved. The proof of (4.2) is similar, and we omit it.

We checked that the assumptions of Theorem 3.1 hold for the processes $X_{n}$ and $X$ and for functionals $\varphi_{n}$ defined by (1.1)-(1.4). Therefore Theorem 2.1 is proved.

Proof of Proposition 1.1. The family $\left\{f^{\delta}\right\}$ is monotone in $\delta$. Thus the implication

$$
\text { (i) } \Rightarrow \text { (ii) }
$$

follows from Dini's theorem, since

$$
f^{t}(x) \rightarrow 0, \quad\|x\| \rightarrow+\infty
$$

for any fixed $t \in \mathbb{R}^{+}$in view of $\left.\mathbf{B} 2\right)$ and the boundedness of the support of $\mu$. The implication (ii) $\Rightarrow$ (i) follows from Proposition 4.1 since uniform continuity is preserved in the case of uniform convergence. The implication (iii) $\Rightarrow$ (ii) is a consequence of (4.2). To prove the converse implication we apply property B3) and a reasoning similar to that used above. Namely,

$$
\begin{gathered}
f^{\delta}(x) \geq \int_{\mathbb{R}^{m}} R_{\delta}(\|y-x\|) \mu(d y), \\
R_{\delta}(z) \equiv \int_{0}^{\delta}\left[M_{1} t^{-m / 2} \exp \left(-\frac{\alpha z^{2}}{t}\right)-M_{2} t^{-m / 2+\lambda} \exp \left(-\frac{\beta z^{2}}{t}\right)\right] d t .
\end{gathered}
$$

Changing the variables $u=z^{2} / t$ we get

$$
\begin{gathered}
R_{\delta}(z)=z^{2-m} G_{1}\left(\frac{z^{2}}{\delta}\right)-z^{2-m+2 \lambda} G_{2}\left(\frac{z^{2}}{\delta}\right), \\
G_{1}(y) \equiv M_{1} \int_{y}^{\infty} u^{m / 2-2} \exp [-\alpha u] d u, \quad G_{2}(y) \equiv M_{2} \int_{y}^{\infty} u^{m / 2-2-\lambda} \exp [-\beta u] d u .
\end{gathered}
$$

Without loss of generality we assume that $\lambda<1$. Below we separate two cases, $m \geq 3$ and $m=2$. If $m \geq 3$, then the functions $G_{1}$ and $G_{2}$ are bounded; thus

$$
R_{\delta}(z) \geq z^{2-m}\left[G_{1}(\delta)-\delta^{2 \lambda} G_{2}(\delta)\right] \geq D w(z)
$$

for $z<\delta$ if $\delta$ is sufficiently small. Thus

$$
f^{\delta}(x) \geq D \int_{\|y-x\| \leq \delta} w(\|y-x\|) \mu(d y)
$$

if $\delta$ is sufficiently small. 
If $m=2$, then $G_{1}(y) \sim-\ln y$ as $y \downarrow 0$. Hence

$$
G_{1}\left(\frac{z^{2}}{\delta}\right) \geq D \ln \frac{\delta}{z^{2}} \geq D \ln \frac{1}{z}=D w(z)
$$

for all $z<\delta$ if $\delta$ is sufficiently small. Further, $G_{2}(y) \sim \lambda^{-1} y^{-\lambda}$ as $y \downarrow 0$, whence

$$
z^{2 \lambda} G_{2}\left(\frac{z^{2}}{\delta}\right) \leq D z^{2 \lambda}\left(\frac{\delta}{z^{2}}\right)^{\lambda}=D \delta^{\lambda}
$$

for all $z<\delta$ if $\delta$ is sufficiently small. The latter two bounds imply inequality (4.11) for $m=2$, since $w \geq 1$ if $m=2$. This proves the implication (ii) $\Rightarrow$ (iii). Proposition [1.1] is proved.

\section{EXAMPLES}

We present two examples illustrating Theorem 2.1 in this section. First, we construct a measure $\mu$ that satisfies condition (1.13) but does not satisfy condition (1.11). The $W$-functional corresponding to this measure cannot be obtained as the limit in the framework of the paper [11. On the other hand, this functional belongs to the class of limit functionals considered in Theorem 2.1 (see Remark 2.3).

Example 5.1. Let $m=2$ and let $\left\{r_{k}, k \geq 1\right\}$ be a sequence of positive numbers to be specified later. The measure $\mu$ is defined by $\mu=\sum_{k \geq 1} Q_{k} \mu_{k}$, where $\left\{Q_{k}, k \geq 1\right\}$ is a certain sequence of weights and $\mu_{k}=\delta_{S_{k}}$ is the surface measure in the circumference $S_{k} \equiv\left\{y:\|y\|=r_{k}\right\}, k \geq 1$. The measures $\mu_{k}$ are normalized so that $\mu_{k}\left(S_{k}\right)=1, k \geq 1$.

For an arbitrary $x \in \mathbb{R}^{2}$ and $\delta \in\left(0, e^{-1}\right)$, put $B(x, \delta)=\{y:\|y-x\| \leq \delta\}$. Then

$$
\begin{gathered}
\int_{\|y-x\| \leq \delta} w(\|y-x\|) \mu(d y)=\sum_{k} Q_{k} V_{k}^{\delta}(x), \\
V_{k}^{\delta}(\cdot) \equiv \int_{S_{k} \cap B(x, \delta)} w(\|y-\cdot\|) \mu_{k}(d y) .
\end{gathered}
$$

Up to the factor $(2 \pi)^{-1}, V_{k}^{\delta}$ is the potential of the simple layer generated by the measure $\mathbb{1}_{B(x, \delta)} d \mu_{k}$ on $S_{k}$ (see, for example, [19], $\S 21$ ). This potential is dominated by the potential $V_{k}$ generated by the measure $\mu_{k}$. Both these potentials are continuous functions that are harmonic in $\left\{y:\|y\|<r_{k}\right\}$ and $\left\{y:\|y\|>r_{k}\right\}$. According to the maximum principle for harmonic functions,

$$
\begin{gathered}
V_{k}(z)=V_{k}(0)=\ln \left(\frac{1}{r_{k}}\right), \quad\|z\| \leq r_{k}, \\
V_{k}^{\delta}(x) \leq V_{k}(x) \leq \ln \left(\frac{1}{r_{k}}\right), \quad x \in \mathbb{R}^{2}, \quad \delta \in\left(0, e^{-1}\right),
\end{gathered}
$$

$k \geq 1$. Using the same maximum principle again we prove that $V_{k}^{\delta}$ assumes its maximal value on $S_{k}$. If $\delta<r_{k}$, then $S_{k} \cap B(x, \delta)$ is an arc of the circumference $S_{k}$. It is not hard to check that the corresponding maximal value is attained at the middle point of this arc. Using a simple bound we get

$$
V_{k}^{\delta}(x) \leq D\left(\frac{\delta}{r_{k}}\right) \ln \left(\frac{1}{\delta}\right) .
$$

Now we put $r_{k}=2^{-k^{2}}$ and $Q_{k}=k^{-4}, k \geq 1$. Let $\delta \in\left(0, e^{-1}\right)$ be fixed and let $x \in \mathbb{R}^{m}$ be arbitrary. We estimate $\int_{\|y-x\| \leq \delta} w(\|y-x\|) \mu(d y)$. If $\|x\| \leq 2 \delta$, then $B(x, \delta) \subset B(0,3 \delta)$, 
whence

$$
\int_{\|y-x\| \leq \delta} w(\|y-x\|) \mu(d y) \leq \sum_{k: r_{k} \leq 3 \delta} Q_{k} \ln \left(\frac{1}{r_{k}}\right)=\sum_{k: r_{k} \leq 3 \delta} \frac{1}{k^{2}}
$$

by (5.1). Otherwise, that is, if $\|x\|>2 \delta$, then there exists at most one value $k=k_{*}$ such that

$$
B(x, \delta) \cap S_{k_{*}} \neq \varnothing .
$$

Moreover $r_{k_{*}} \geq \delta$. According to (5.1) and (5.2),

$$
\begin{aligned}
\int_{\|y-x\| \leq \delta} w(\|y-x\|) \mu(d y) & \leq D \sup _{k: r_{k}>\delta}\left[\ln r_{k}\right]^{-2}\left(\frac{\delta}{r_{k}}\right) \ln \left(\frac{1}{\delta}\right) \\
& =D \sup _{k: r_{k}>\delta} \frac{\varphi(\delta)}{\varphi\left(r_{k}\right)}\left[\ln \left(\frac{1}{\delta}\right)\right]^{-1},
\end{aligned}
$$

where $\varphi(t)=t[\ln t]^{2}$ and the function $\varphi(\cdot)$ is nondecreasing in some neighborhood to the right of the origin. The following estimate follows from (5.3) and (5.4):

$$
\sup _{x \in \mathbb{R}^{m}} \int_{\|y-x\| \leq \delta} w(\|y-x\|) \mu(d y) \leq D\left[\ln \left(\frac{1}{\delta}\right)\right]^{-1 / 2} .
$$

In its turn, the latter estimate implies (1.13). On the other hand,

$$
\mu\left(B\left(0, r_{k}\right)\right)>Q_{k}=\left(\frac{1}{\ln r_{k}}\right)^{2} .
$$

Thus

$$
\limsup _{r \downarrow 0} r^{-\gamma} \mu(B(0, r))=+\infty
$$

for an arbitrary $\gamma>0$ which means that (1.11) does not hold.

In the second example, we construct a $W$-measure $\mu$ that does not satisfy condition (1.13). The $W$-functional corresponding to this measure cannot be obtained as the limit in Theorem 2.1.

Example 5.2. Let $m=2, r_{k}=2^{-k^{2}}, a_{k}=(1 / k, 0) \in \mathbb{R}^{m}, \tilde{S}_{k}=\left\{y:\left\|y-a_{k}\right\|=r_{k}\right\}$, $\tilde{Q}_{k}=k^{-2}, k \geq 1$, and let $\tilde{\mu}_{k}=\delta_{\tilde{S}_{k}}$ be the surface measure on the circumference $\tilde{S}_{k}$ normalized so that $\tilde{\mu}_{k}\left(\tilde{S}_{k}\right)=1, k \geq 1$. Put

$$
\tilde{\mu}=\sum_{k} \tilde{Q}_{k} \tilde{\mu}_{k} .
$$

We show that $\tilde{\mu}$ is a $W$-measure that does not satisfy condition (1.13). By construction, there exists $N \in \mathbb{N}$ such that the relation

$$
\left\|x-a_{k}\right\| \leq 2^{-\sqrt{k}}
$$

holds for at most $N$ values of $k \in \mathbb{N}$ whatever $x \in \mathbb{R}^{m}$ is. Moreover, if $k>4$ and (5.5) holds, then $\|y-x\| \leq e^{-1}$ for $y \in \tilde{S}_{k}$. Thus, given $x \in \mathbb{R}^{m}$, we again apply the maximum principle and obtain the following bound:

$$
\begin{aligned}
& \tilde{Q}_{k} \int_{\|y-x\| \leq 1} w(\|y-x\|) \tilde{\mu}_{k}(d y) \leq \tilde{Q}_{k} \int_{\mathbb{R}^{2}} w(\|y-x\|) \tilde{\mu}_{k}(d y) \\
& \quad \leq \tilde{Q}_{k} \int_{\mathbb{R}^{2}} w\left(\left\|y-a_{k}\right\|\right) \tilde{\mu}_{k}(d y)=\tilde{Q}_{k} \int_{\tilde{S}_{k}} \ln \left(\frac{1}{\left\|y-a_{k}\right\|}\right) \tilde{\mu}_{k}(d y) \\
& \quad=\tilde{Q}_{k} \ln \left(\frac{1}{r_{k}}\right)=\tilde{Q}_{k} k^{2}=1
\end{aligned}
$$


for those $k>4$ that satisfy (5.5). If $k>1$ and (5.5) does not hold, then

$$
\|y-x\| \geq 2^{-\sqrt{k}-1}, \quad y \in \tilde{S}_{k} .
$$

Thus, given $x \in \mathbb{R}^{m}$, we obtain the estimate

$$
\tilde{Q}_{k} \int_{\|y-x\| \leq 1} w(\|y-x\|) \tilde{\mu}_{k}(d y) \leq \tilde{Q}_{k}(\sqrt{k}+1)=k^{-3 / 2}+k^{-2}
$$

for those $k>4$ that satisfy (5.5). Moreover, every measure $\tilde{\mu}_{k}$ is a $W$-measure; hence

$$
\sigma_{k} \equiv \sup _{x \in \mathbb{R}^{m}} \int_{\|y-x\| \leq 1} w(\|y-x\|) \tilde{\mu}_{k}(d y)<+\infty, \quad k \geq 1 .
$$

The latter three estimates imply that

$$
\sup _{x \in \mathbb{R}^{m}} \int_{\|y-x\| \leq 1} w(\|y-x\|) \tilde{\mu}(d y) \leq \sum_{k=1}^{4} k^{-2} \sigma_{k}+N+\sum_{k \geq 5}\left(k^{-3 / 2}+k^{-2}\right)<+\infty,
$$

that is, $\tilde{\mu}$ is a $W$-measure. On the other hand,

$$
\int_{\left\|y-x_{k}\right\| \leq \delta_{k}} w\left(\left\|y-x_{k}\right\|\right) \tilde{\mu}(d y) \geq \tilde{Q}_{k} \int_{\left\|y-x_{k}\right\| \leq \delta_{k}} w\left(\left\|y-x_{k}\right\|\right) \tilde{\mu}_{k}(d y)=1
$$

for $\delta_{k}=r_{k}$ and $x_{k}=a_{k}$. Thus $\delta_{k} \rightarrow 0$ and $\tilde{\mu}$ does not satisfy condition (1.13).

\section{BIBLIOGRAPHY}

1. A. V. Skorokhod, Asymptotic Methods in the Theory of Stochastic Differential Equations, Naukova Dumka, Kiev, 1987; English transl., AMS, Providence, 1992. MR913305 (88m:60164) MR 1020057 (90i:60038)

2. E. B. Dynkin, Markov Processes, Fizmatgiz, Moscow, 1963; English transl., Springer-Verlag, Berlin-Göttingen-Heidelberg, 1965. MR0193670 (33:1886), MR0193671(33:1887)

3. A. V. Skorokhod and N. P. Slobodenyuk, Limit Theorems for Random Walks, Naukova Dumka, Kiev, 1970. (Russian) MR0282419 (43:8130)

4. A. N. Borodin and I. A. Ibragimov, Limit Theorems for Functionals of Random Walks, Proceedings of Mathematical Institute of RAN, vol. 195, Nauka, Sankt Petersburg, 1994; English transl., AMS, Providence, RI, 1995. MR1368394 (97j:60140)

5. I. I. Gikhman, Some limit theorems on the number of intersections of a random function and the boundary of a given domain, Nuk. Zap. Kiev University 16 (1957), no. 10, 149-164. (Ukrainian)

6. I. I. Gikhman, Asymptotic distributions of the number of intersections of a random function and the boundary of a given domain, Visnyk Kiev University, Ser. Astronom, Matem., Mekh. 1 (1958), no. 1, 25-46. (Ukrainian)

7. N. I. Portenko, Integral equations and limit theorems for additive functionals of a Markov process, Teor. Veroyatnost. i Primenen. 12 (1967), no. 3, 551-558; English transl. in Theory Probab. Appl. 12 (1967), no. 3, 500-505. MR0221596 (36:4648)

8. A. N. Borodin, On the asymptotic behavior of local times of recurrent random walks with finite variance, Teor. Veroyatnost. i Primenen. 26 (1981), no. 4, 769-783; English transl. in Theory Probab. Appl. 26 (1981), no. 4, 758-772. MR636771 (83a:60120)

9. E. Perkins, Weak invariance principles for local time, Z. Wahrscheinlichkeitstheorie verw. Gebiete 60 (1982), 437-451. MR665738 (83h:60080)

10. A. S. Cherny, A. N. Shiryaev, and M. Yor, Limit behavior of the "horizontal-vertical" random walk and some extensions of the Donsker-Prokhorov invariance principle, Teor. Veroyatnost. i Primenen. 47 (2002), no. 3, 498-517; English transl. in Theory Probab. Appl. 47 (2003), no. 3, 377-394. MR1975425 (2004b:60121)

11. R. F. Bass and D. Khoshnevisan, Local times on curves and uniform invariance principles, Prob. Theory Related Fields 92 (1992), 465-492. MR1169015(93e:60161)

12. E. B. Dynkin, Self-intersection gauge for random walks for Brownian motion, Ann. Probab. 16 (1988), 1-57. MR920254 (89f:60053)

13. V. Konakov and E. Mammen, Local limit theorems for transition densities of Markov chains converging to diffusions, Prob. Theory Related Fields 117 (2000), 551-587. MR1777133 (2001j:60141) 
14. V. Konakov, Small Time Asymptotics in Local Limit Theorems for Markov Chains Converging to Diffusions, arXiv:math. PR/0602429, 2006.

15. Yu. N. Kartashov and A. M. Kulik, Weak convergence of additive functionals of a sequence of Markov chains, Theory Stoch. Process. 15(31) (2009), no. 1. (to appear)

16. A. M. Kulik, Markov approximation of stable processes by random walks, Theory Stoch. Proccess. 12(28) (2006), no. 1-2 87-93. MR2316289 (2008j:60082)

17. A. S. Gibbs and F. E. Su, On choosing and bounding probability metrics, Internat. Statist. Review 70 (2001), 419-35.

18. J. L. Doob, Stochastic Processes, John Wiley, New York, 1953. MR0058896 (15:445b)

19. V. S. Vladimirov, Equations of Mathematical Physics, Nauka, Moscow, 1967; English transl., Marcel Dekker, New York, 1971. MR.0239242(39:599) MR0268497(42:3394)

Institute of Mathematics, National Academy of Sciences of Ukraine, Tereshchenkivs'ka Street 3, 01601, Kyiv, Ukraine

E-mail address: kulik@imath.kiev.ua

Received 12/FEB/2007

Translated by S. KVASKO 